\title{
561 TRIPLE CHECKPOINT BLOCKADE, BUT NOT ANTI-PD1 ALONE, ENHANCES THE EFFICACY OF ENGINEERED ADOPTIVE T CELL THERAPY IN ADVANCED OVARIAN CANCER
}

Kristin Anderson*, Yapeng Su, Madison Burnett, Breanna Bates, Magdalia Rodgers Suarez, Susan Ruskin, Aesha Vakil, Valentin Voillet, Raphael Gottardo, Philip Greenberg. Fred Hutchinson Cancer Research Center, Seattle, WA, USA

Background Over 20,000 women are diagnosed with ovarian cancer annually, and more than half will die within 5 years. This rate has changed little in the last 20 years, highlighting the need for therapy innovation. A promising new strategy with the potential to control tumor growth without toxicity to healthy tissues employs immune $\mathrm{T}$ cells engineered to target proteins uniquely overexpressed in tumors. Mesothelin (Msln) contributes to the malignant and invasive phenotype in ovarian cancer, and has limited expression in healthy cells, making it a candidate immunotherapy target in these tumors.

Methods The ID8 ${ }_{\text {VEGF }}$ mouse cell line was used to evaluate if $\mathrm{T}$ cells engineered to express a mouse Msln-specific high-affinity $\mathrm{T}$ cell receptor (TCRMsln) can kill murine ovarian tumor cells in vitro and in vivo. Tumor-bearing mice were treated with $\mathrm{TCR}_{\mathrm{Msln}} \mathrm{T}$ cells plus anti-PD-1, anti-Tim-3 or anti-Lag-3 checkpoint-blocking antibodies administered alone or in combination, ultimately allowing targeting up to three inhibitory receptors simultaneously. Single cell RNA sequencing was used to profile the impact of combination checkpoint blockade on both the engineered $\mathrm{T}$ cells and the tumor microenvironment. Results In a disseminated ID8 tumor model, adoptively transferred $\mathrm{TCR}_{\mathrm{Msln}} \mathrm{T}$ cells preferentially accumulated within established tumors, delayed ovarian tumor growth, and significantly prolonged mouse survival. However, our data also revealed that elements in the tumor microenvironment (TME) limited engineered $\mathrm{T}$ cell persistence and ability to kill cancer cells. Triple checkpoint blockade, but not single- or double-agent treatment, dramatically increased anti-tumor function by intratumoral $\mathrm{TCR}_{\mathrm{M} \text { sin }} \mathrm{T}$ cells. Single cell RNA-sequencing revealed distinct transcriptome changes in engineered $\mathrm{T}$ cells and the TME following triple blockade compared to single- and double-agent treatment. Moreover, combining adoptive immunotherapy with triple checkpoint blockade prolonged survival in the cohort of treated tumor-bearing mice, relative to $\mathrm{TCR}_{\mathrm{Msln}}$ with or without anti-PD1, or double-agent treatments.

Conclusions Inhibitory receptor/ligand interactions within the tumor microenvironment can dramatically reduce $\mathrm{T}$ cell function, suggesting tumor cells may evade $\mathrm{T}$ cell responses by upregulating the ligands for PD-1, Tim-3 and Lag-3. In a model of advanced ovarian cancer, triple checkpoint blockade significantly improved the function of transferred engineered $\mathrm{T}$ cells and improved outcomes in mice in a setting in which single checkpoint blockade had no significant activity. The results suggest that $\mathrm{T}$ cell therapy with triple blockade, which can ultimately be more safely pursed in a cell intrinsic form through $\mathrm{T}$ cell genetic engineering, may overcome barriers to achieving therapeutic efficacy in patients.

Ethics Approval The Institutional Animal Care and Use Committees of the University of Washington and the Fred Hutchinson Cancer Research Center approved all animal studies.

http://dx.doi.org/10.1136/jitc-2021-SITC2021.561 